\title{
Isolation of Mucorales from biological environment and identification of Rhizopus among the isolates using PCR- RFLP
}

\author{
Mahboobeh Madani ${ }^{*}{ }^{\circledR}$, Mohammadali Zia $^{2}$ \\ ${ }^{1}$ Department of Microbiology, Falavarjan Branch, Islamic Azad University, Isfahan, Iran \\ ${ }^{2}$ Department of Basic Science, (Khorasgan) Isfahan Branch, Islamic Azad University, Isfahan, Iran
}

*Corresponding Author: Mahboobeh Madani, Tel: + 989134097629, Email: mmadani66@gmail.com

\begin{abstract}
Background and aims: Mucorales are fungi belonging to the category of Zygomycetes, found much in nature. Culture-based methods for clinical samples are often negative, difficult and time-consuming and mainly identify isolates to the genus level, and sometimes only as Mucorales. Therefore, applying fast and accurate diagnosis methods such as molecular approaches seems necessary. This study aims at isolating Mucorales for determination of Rhizopus genus between the isolates using molecular methods.

Methods: In this descriptive observational study, a total of 500 samples were collected from air and different surfaces and inoculated on Sabouraud Dextrose Agar supplemented with chloramphenicol. Then, the fungi belonging to Mucorales were identified and their pure culture was provided. DNA extraction was done using extraction kit and the chloroform method. After amplification, the samples belonging to Mucorales were identified by observing 830 bp bands. For enzymatic digestion, enzyme BmgB1 was applied for identification of Rhizopus species by formation of 593 and 235 bp segments.

Results: One hundred pure colonies belonging to Mucorales were identified using molecular methods and after enzymatic digestion, 21 isolates were determined as Rhizopus species. The sequencing of PCR products and macroscopic and microscopic studies confirmed the existence of $R$. stolonifera, $R$. oryzae and $R$. caespitosus in the samples.

Conclusion: Generally, developing a reliable method for determining Zygomycete species can be a useful tool for better understanding of the epidemiology of mucoromycosis.

Keywords: Mucorales, Rhizopus, PCR-RFLP, Isolation, Environment
\end{abstract}

Received: 17 October 2017, Accepted: 6 March 2019, ePublished: 4 April 2019

\section{Introduction}

Invasive fungal infections are major medical complications in immunocompromised patients. In cases such as cancer and organ transplantation, invasive fungal infections are of particular importance.

Although aspergillosis and candidiasis are among the most common invasive fungi, there is evidence that the presence of Zygomycetes infections has dramatically increased in the past decade (1-4). According to the latest study, mucormycosis in patients receiving bone marrow hematopoietic cells is the most common fungal infection after candidiasis and aspergillosis.

Failure to histopathologically detect and confirm mucormycosis is one of the important outcomes of treatment. In comparison, Aspergillus species are mainly susceptible to voriconazole and amphotericin B and Mucorales only to amphotericin B and to a lesser extent posaconazole (5). In addition, the identification of Zygomycetes using morphological features alone is problematic and time-consuming and requires examination by experienced people (6-9).

Zygomycetes usually grow in a standard growth medium such as a Sabouraud Dextrose Agar, and most of the samples isolated from humans grow in this fast-growth environment and cover the whole plate within 2-3 days.

Microscopically, Mucorales have wide aseptate and transparent mycelia $(9,10)$.

Due to the disadvantages and problems of the identification of Zygomycetes using the microbiological and histopathological methods, it is obviously necessary to use molecular methods $(5,9,10)$.

Diagnosis of zygomycosis is difficult, and recent advances in the study of new molecular methods have been made.

Two major issues, i.e., identification of species from different isolated strains from the culture and identification of Zygomycetes are taken into account in clinical specimens.

Multiple molecular targets and increased DNA sequences accessible in international databases have shown that accurate identification of Zygomycetes is possible.

(c) 2019 The Author(s); Published by Shahrekord University of Medical Sciences. This is an open-access article distributed under the terms of the Creative Commons Attribution License (http://creativecommons.org/licenses/by/4.0), which permits unrestricted use, distribution, and reproduction in any medium, provided the original work is properly cited. 
In any case, the cultivation result of clinical specimens is often negative and their appearance is the same in histological techniques. In addition, it is often difficult to differentiate a Zygomycetes from other hyphomycetes. Therefore, the replacement of molecular detection methods to determine the zygomycosis and the identification of species directly from the culture or tissue are essential (7).

Polymerase chain reaction (PCR) is perhaps one of the most important and useful methods. PCR-based methods save time and are highly specific and sensitive.

Common molecular targets are to determine and quickly detect fungal pathogens in sequences within the rDNA gene. This part of the gene contains variable DNA sequences from ITS regions. Common molecular methods based on ITS regions for the identification of fungi include the use of primers and probe specific to the genus or species, restriction fragment length polymorphism (RFLP) for DNA replication, or direct analysis of the DNA sequence of the reproduction. It has been shown that ITS regions are highly capable of being used in molecular methods as a target to identify fungal species (6).

One of the advantages of molecular detection method is high reliability, which indicates the superiority of this method over other methods (11).

The purpose of this study was to isolate Mucorales from the environment and determine the Rhizopus genus using PCR-RFLP. In addition, the study of the prevalence of Mucorales in different cultures, the study of the prevalence of Rhizopus species in different cultures, the study of the use of molecular methods in the determination and detection of Mucorales, and an investigation on feasibility of using molecular method in the determination and identification of Rhizopus were taken into account in this study.

Molecular techniques have demonstrated a potential ability for quick and accurate diagnosis of mucormycosis, and the development of these techniques is particularly important to detect these fungi.

\section{Materials and Methods}

In this descriptive study during 2012-2015, 500 samples were collected from different environments, including air, soil, organic decomposing materials, and foodstuffs such as fruits, seeds, and bread.

Air samples were collected using an open-plate method at an approximate height of one meter from the ground for 30 to 60 minutes. Surface sampling (in hospitals, fruit stores, bakeries, etc) was conducted using a sterile swab, foodstuff sampling by cutting a piece of the foodstuff, and soil sampling in a depth of $5-10 \mathrm{~cm}$ in soil using sterile metal spoons.

All samples were transferred to the Mycology Laboratory of Islamic Azad University, Falavarjan Branch, and cultured in a Sabouraud Dextrose Agar containing chloramphenicol. First, all cultured samples were purified and then the identification of fungi was carried out based on the morphologic and microscopic features of colonies. Fungi were identified at the genus and, if possible, species levels.

The suspension was prepared from all strains isolated from fungal spores in sterile distilled water and stored at $-20^{\circ} \mathrm{C}$ until use, and if necessary, inoculated in a Sabouraud Dextrose Agar containing chloramphenicol and incubated at $30^{\circ} \mathrm{C}$.

The genera belonging to the Mucorales order usually have rapid growth and mycelium elements cover the full surface of the Petri dish within 2 to 3 days after incubation.

The vegetative mycelia of all Mucorales species are composed of transparent, wide, and transverse wall lacking hives.

The common growth characteristics used to differentiate Mucorales include colony morphology, the presence of sporangiophores, Sporangia, and the presence or absence of rhizoids (9).

All samples were purified and the DNA was extracted using the QIA using the QIAamp DNA Mini Kit (QIAGEN) and the High Pure PCR Template Preparation Kit (Roche Diagnosis, Meylan, France). Approximately $50 \mathrm{mg}$ of the mycelia of the crashed fresh fungal culture (without sporangium) was suspended in $700 \mu \mathrm{L}$ of CTAB extraction buffer (100 mM Tris- $\mathrm{Cl}$ [pH 8.4], $1.4 \mathrm{M} \mathrm{NaCl}$, $25 \mathrm{mM}$ EDTA, 2\% CTAB) and the resulting solution was vortexed for 10 seconds, an equal volume of chloroform was added to the microtube, and the resulting solution was vortexed for 5 seconds and microcentrifuged at 12300 rpm for 10 minutes. Then, $500 \mu \mathrm{L}$ of the supernatant was removed and transferred to a new $1.5 \mathrm{~mL}$ microtube, and the DNA sedimentation was obtained by adding an equal volume of 2-propanol and microcentrifugation at 12300 rpm for 1 minute, so that the sedimentation formed at the bottom of the microtube. The supernatant and sedimentation were washed slowly with $70 \%$ ethanol and resuspended in $200 \mu \mathrm{L}$ of TE buffer $(10 \mathrm{mM}$ Tris- $\mathrm{Cl}[\mathrm{pH}$ 8.0], $1 \mathrm{mM}$ EDTA [pH 8.0]). For amplification of PCR, $8 \mu \mathrm{L}$ of stock DNA was diluted in $1 \mathrm{~mL}$ of deionized distilled water and stored at $-20^{\circ} \mathrm{C}$ until use (12).

Selected sites for designing of primers were adjusted based on the software available at http://www.genebee.msu.su/ genebee.html, and short oligonucleotide sequences were used for amplification of the major isolated Mucorales (in the $18 \mathrm{~S}$ rRNA region) designed by Machouart et al (4).

The primer (MR1: 5 'AGTAGTTTGTCTTCGGTCAA 3 ') was used to determine the Mucorales sequence and the specificprimer(RpL1:5'TGATCTACGTGACAAATTCT 3') to determine the Rhizopus genus, which bind to the sites 75 and 901, respectively, in the regions of the template (4).

The following materials were used to prepare each sample for conducting PCR.

Two microliters (equivalent to a few $\mu \mathrm{g}$ ) of the DNA template of each sample was added to $12.5 \mu \mathrm{L}$ of Taq DNA Polymerase (Amplicon, Denmark), $0.5 \mu \mathrm{L}$ of each 
primer (equivalent to few $\mathrm{pM} / \mathrm{L}$ ), and $8 \mu \mathrm{L}$ of sterile distilled water that had been distilled two times, so that the total volume of the reaction reached $25 \mu \mathrm{L}$.

DNA molecules belonging to the Mucorales were amplified using a selected primer and amplified an approximate 830 bp band for Mucorales.

The PCR product obtained in $1.5 \%$ agarose gel was detected by ethidium bromide under UV lamp. A negative control sample (without DNA template) was used to identify any contamination in the reaction. The PCR program was performed as follows:

Primary Denaturation: 5 minutes, $94^{\circ} \mathrm{C}$

Denaturation: 1 minute, $94^{\circ} \mathrm{C}, 30$ cycles

Annealing: 1 minute, $60^{\circ} \mathrm{C}, 30$ cycles

Extension: 1 minute, $72^{\circ} \mathrm{C}, 30$ cycles

Final Extension: 5 minutes, $72^{\circ} \mathrm{C}$

Determination of the isolates of the Rhizopus genus belonging to the Mucorales was based on the observation of 830 bp band.

The $B m g B$ I enzyme was used specifically to determine and isolate different species of Rhizopus genus, and the sizes of the resulting fragments were estimated after enzymatic digestion in $2 \%$ gel electrophoresis using the trans-illuminator. The restriction site of the $B m g B I$ enzyme was (5 'CACGTC 3') and the sizes of the fragments were $593 \mathrm{bp}$ and $235 \mathrm{bp}$ (Figures 1 and 2). This enzyme is used to identify Rhizopus species other than Rhizopus stolonifer (4). In order to confirm the detection, and in particular to determine $R$. stolonifer, sequencing of the PCR product was also performed. Sequencing was performed using the Geneious software. The fragments obtained from the studied colonies were placed on an agarose gel after PCR to determine their purity and relative size, so that after sequencing and matching on the NCBI website, their homology and overlapping with corresponding strains were reviewed.

\section{Results}

A total of 346 pure colonies were obtained. After microscopic and macroscopic examinations, 100 colonies

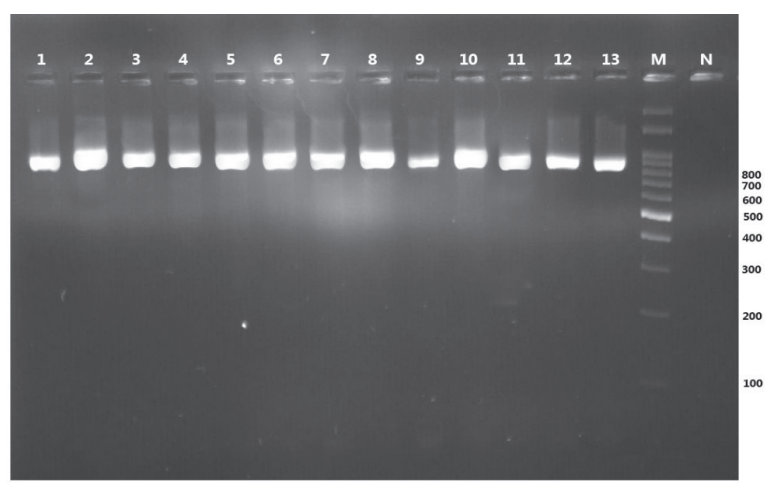

Figure 1. Agarose gel electrophoresis of $18 \mathrm{~S}$ rRNA PCR products of Mucorales. A single band of approximately 830 bp was seen in lanes of 1 to 13; Lane M: Molecular marker and Lane N: Negative control.

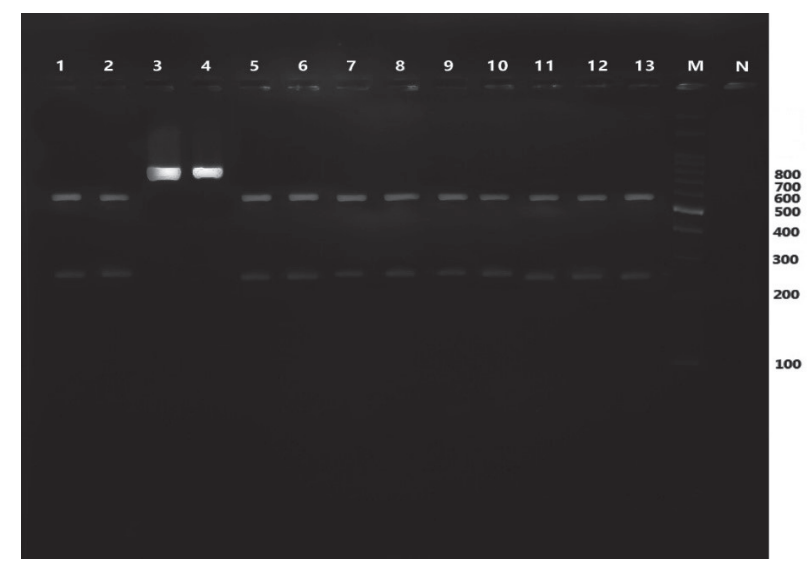

Figure 2. Agarose gel electrophoresis of $18 \mathrm{~S}$ rRNA PCR products of Mucorales after enzymatic digestion with BmgBI enzyme. Lanes 1, 2 and 5 to 13: Rhizopus sp; Lanes 3 and 4: undigested PCR products; Lane M: Molecular marker and Lane $\mathrm{N}$ : negative control

belonging to the Mucorales were purified and isolated (Table 1).

Subsequently, their belonging to the Mucorales sequence and the Rhizopus genus was confirmed by specific primers. $B m g B$ I enzyme was used to isolate the species belonging to the Rhizopus genus.

As a result, 21 colonies were identified as species belonging to the Rhizopus genus. Out of $21 \mathrm{PCR}$ products that were sent for sequencing, 15 cases $(71.43 \%)$ were identified as $R$. stolonifer, 3 cases $(14.29 \%)$ as Rhizopus oryzae, 1 case $(4.76 \%)$ as Rhizopus caespitosus, and 2 (9.52\%) as unspecified species of Rhizopus (Table 2).

\section{Discussion}

Regarding the importance of zygomycosis infections

Table 1. Variety distribution of isolated fungi from environment

\begin{tabular}{|c|c|c|c|c|}
\hline \multirow{2}{*}{ Genus } & \multicolumn{2}{|c|}{ Colonies } & \multirow{2}{*}{ Total } & \multirow{2}{*}{$\%$} \\
\hline & Air & Surfaces & & \\
\hline Acrophialophora & 0 & 1 & 1 & 0.28 \\
\hline Alternaria & 32 & 28 & 60 & 17.34 \\
\hline Aspergillus & 26 & 38 & 64 & 18.50 \\
\hline Aureobasidium & 2 & 5 & 7 & 2.02 \\
\hline Chrysosporium & 3 & 1 & 4 & 1.16 \\
\hline Cladosporium & 7 & 11 & 18 & 5.20 \\
\hline Curvularia & 5 & 1 & 6 & 1.73 \\
\hline Drechslera & 9 & 2 & 11 & 3.18 \\
\hline Fusarium & 3 & 1 & 4 & 1.16 \\
\hline Paecilomyces & 1 & 2 & 3 & 0.87 \\
\hline Penicillium & 29 & 27 & 56 & 16.18 \\
\hline Scopulariopsis & 3 & 2 & 5 & 1.45 \\
\hline Stemphylium & 2 & 2 & 4 & 1.16 \\
\hline Ulocladium & 3 & 0 & 3 & 0.87 \\
\hline Mucorales & 56 & 44 & 100 & 28.90 \\
\hline Total & 181 & 165 & 346 & 100 \\
\hline
\end{tabular}


Table 2. Obtained results from PCR; Sequencing and sequence alignment using Geneious software

\begin{tabular}{|c|c|c|c|c|}
\hline Recognized species & Sequence registration number & Matching rate & Length of the sequenced fragment & Number of colonies \\
\hline Rhizopus oryzae 8-3M & KF717370.1 & $100 \%$ & 211 & R13 \\
\hline Rhizopus stolonifer KACC 45890 & JN315028.1 & $96 \%$ & 317 & R6 \\
\hline Rhizopus stolonifer R-176 & DQ641317.1 & $97 \%$ & 300 & R10 \\
\hline Rhizopus stolonifer GL8921 & JX241668.1 & $99 \%$ & 289 & R11 \\
\hline Rhizopus sp. UASWS0183 & DQ195064.1 & $97 \%$ & 251 & R15 \\
\hline Rhizopus stolonifer KACC 45890 & JN315028.1 & $97 \%$ & 318 & R8 \\
\hline Rhizopus stolonifer R-176 & DQ641317.1 & $98 \%$ & 299 & R14 \\
\hline Rhizopus stolonifer KACC 46105 & JN315031.1 & $97 \%$ & 202 & R15 \\
\hline Rhizopus caespitosus CBS 427.87 & DQ641325.1 & $94 \%$ & 463 & R7 \\
\hline
\end{tabular}

in humans and animals, and considering the problems and disadvantages of morphological and histopathologic diagnosis and also considering the advantages of using molecular methods, the necessity of using and developing these methods to accurately and rapidly identify its causes becomes more pronounced.

One of the advantages of molecular detection methods is high reliability that indicates the superiority of this method over other methods (11).

Although immunological methods have been developed to identify $R$. arrhizus and Basidiobolus species, crossreaction of antibodies is often observed. Despite these problems, DNA-based molecular typing methods have shown a high potential for rapid and accurate identification of causative agents of zygomycosis (12).

PCR-RFLP is a reliable and easily workable method that targets the $18 \mathrm{~S}$ ribosomal genes of Zygomycetes on DNA extracted from the specimens and thus allows quick and accurate detection of them (10).

In the present study, this method was used based on the targeting of the $18 \mathrm{~S}$ ribosomal Zygomycetes gene to identify species belonging to the Rhizopus genus from pure culture media. However, specific primers can be used for other genera and restriction enzymes to differentiate species $(4,13)$.

In this study, after the cutting of PCR products by the $B m g B$ I enzyme, all species belonging to the Rhizopus genus produced 235 and 593 bp fragments, and after RFLP, in a number of cases, only one $830 \mathrm{bp}$ band was observed which was detected and confirmed as $R$. stolonifer after sequencing. Therefore, it seems that the PCR products of $R$. stolonifer are not digested in the vicinity of the enzyme and only 830 bp will be created.

Mucormycosis is produced by 6 families of Mucorales, but the members of the Mucoraceae family, including Rhizopus, Mucor, Absidia, and Rhizomucor have been frequently isolated from human infections (14).

The Rhizopus genus members are the most common isolates from clinical specimens, and $R$. arrhizus (oryzae) is recognized as the most common cause of human infection and is more common than others, followed by the members of the Mucoraceae family $(15,16)$.

In the present study, $21 \%$ of pure colonies of Mucorales belonged to the Rhizopus genus and 79\% to other genera

Park et al examined genetic affinities and variation in the Rhizopus genus using denaturing gradient gel electrophoresis (DGGE) and PCR-RFLP methods in the rDNA ITS ITS1, ITS2, and 5.8S regions, and reported the similarity range among 20 strains to be $42 \%-100 \%$ by PCR-RFLP (17).

Chakrabarti et al used PCR-RFLP with specific primers and restriction enzymes to detect Apophysomyces elegans (18).

Rickerts et al compared the results of histological methods, culture, and PCR techniques in the diagnosis of invasive fungal infections in clinical specimens such as aspergillosis and zygomycosis, and concluded that PCRbased methods were reliable for the identification of etiologic factors and superior over other methods (11).

Machouart et al examined 36 strains of the main opportunistic Mucorales by the PCR-RFLP method, and isolated and identified different species of Rhizopus, Mucor, Rhizomucor, and Absidia species using distinct, specific primers and enzymes (4). The reliability of this method was confirmed by bioinformatics and fungal cultures.

Hammond et al conducted a study on molecular methods for the diagnosis and identification of mucormycosis, and in some cases, the cultures were negative, so the PCR method is suitable for the confirmation and diagnosis of mycosis (5).

Iwen et al reviewed the methods for identifying Zygomycetes with an emphasis on the advances in molecular diagnosis. They analyzed the diagnostic profile of the Zygomycetes using molecular methods (9).

Nyilasi et al also used PCR methods with sequencing to detect Zygomycetes, and suggested these methods were appropriate to identify species (19). In this study, PCR-RFLP method was used to determine the Rhizopus 
genus among other genera of Mucorales species. Then, sequencing method was used to determine the species of Rhizopus, which identified three species: $R$. stolonifer, $R$. oryzae, and $R$. caespitosus. According to the study of sources regarding the isolation of Mucorales, it seems that the isolation and identification of $R$. caespitosus is rarely reported in Iran (19).

Shakeri et al morphologically studied microbial infections in hairdressers of Shahrekord in a descriptiveanalytical study. Results showed that Staphylococcus aureus was the cause of $12.4 \%$ of microbial infections Among fungal infections, less than $1 \%$ of fungi were dermatophytes (Microsporum nanum), 8.2\% belonged to Candida albicans followed by Penicillium (11\%), Rhizopus (3.3\%), and Scopulariopsis (less than 1\%) (20).

Hoshyar et al conducted a descriptive study of air and surfaces of books and shelves of Shahrekord University of Medical Sciences libraries for the presence of various fungi. A total of 307 samples were collected from airborne fungi and various surfaces of the library, including the Persian and Latin book reservoir, study hall, circulation desk, and journals section. Based on culture results and direct lam, the genus of fungi was determined. Their findings showed that the frequency distribution of fungal growth in Latin books was greater than that in Persian books, shelves, and spaces. The most common type of fungus in different parts of the study was Penicillium (21).

In the present study, the highest frequency among fungi isolated from different environments was obtained for Mucorales (28.98\%), followed by Aspergillus (18.5\%), Alternaria (17.34\%), and Penicillium (18.6\% 16), with differences and similarities with the above-cited studies, which seems to be related to different environmental conditions in different locations.

The isolation of more Mucorales than other fungi seems to be partly related to the fact that some of the samples in this study were obtained from the hospital environment and surfaces. In addition, the time of sampling may lead to different results.

\section{Conclusion}

In this study, the PCR method was used to identify Mucorales strains. Developing a reliable method for the identification of Zygomycetes species can be a useful tool for a better understanding of the epidemiology of mucormycosis.

Conflict of interests

None.

Ethical considerations

The study protocol was approved by the Research Ethics Committee of Khorasgan (Isfahan) Branch, Islamic Azad University, Isfahan, Iran (code: IR.IAU.KHUISF. REC.1397-47).

Acknowledgments

This research was derived from a research project approved at
Islamic Azad University, Falavarjan Branch (code 51721891003001 on $26 / 11 / 89$ ). Hereby, the authors appreciate all those who assisted in conducting this study.

\section{References}

1. Chayakulkeeree M, Ghannoum MA, Perfect JR. Zygomycosis: the re-emerging fungal infection. Eur J Clin Microbiol Infect Dis. 2006;25(4):215-29. doi: 10.1007/s10096-006-0107-1.

2. Gomes MZ, Lewis RE, Kontoyiannis DP. Mucormycosis caused by unusual mucormycetes, non-Rhizopus, -Mucor, and -Lichtheimia species. Clin Microbiol Rev. 2011;24(2):411-45. doi: 10.1128/cmr.00056-10.

3. Kauffman CA. Zygomycosis: reemergence of an old pathogen. Clin Infect Dis. 2004;39(4):588-90. doi: 10.1086/422729.

4. Machouart M, Larche J, Burton K, Collomb J, Maurer P, Cintrat $A$, et al. Genetic identification of the main opportunistic Mucorales by PCR-restriction fragment length polymorphism. J Clin Microbiol. 2006;44(3):805-10. doi: 10.1128/ jcm.44.3.805-810.2006.

5. Hammond SP, Bialek R, Milner DA, Petschnigg EM, Baden LR, Marty FM. Molecular methods to improve diagnosis and identification of mucormycosis. J Clin Microbiol. 2011;49(6):2151-3. doi: 10.1128/jcm.00256-11.

6. De Marco D, Perotti M, Ossi CM, Burioni R, Clementi M, Mancini N. Development and validation of a molecular method for the diagnosis of medically important fungal infections. New Microbiol. 2007;30(3):308-12.

7. Dannaoui E. Molecular tools for identification of Zygomycetes and the diagnosis of zygomycosis. Clin Microbiol Infect. 2009;15 Suppl 5:66-70. doi: 10.1111/j.14690691.2009.02983.x.

8. Alvarez E, Sutton DA, Cano J, Fothergill AW, Stchigel A, Rinaldi MG, et al. Spectrum of zygomycete species identified in clinically significant specimens in the United States. J Clin Microbiol. 2009;47(6):1650-6. doi: 10.1128/jcm.00036-09.

9. Bastola D, Thapa I, Iwen PC. Review of methods for the identification of zygomycetes with an emphasis on advances in molecular diagnostics. Lab Med. 2011;42(5):260-6. doi: 10.1309/LMJ8Z0QPJ8BFVMZF.

10. Walsh TJ, Gamaletsou MN, McGinnis MR, Hayden RT, Kontoyiannis DP. Early clinical and laboratory diagnosis of invasive pulmonary, extrapulmonary, and disseminated mucormycosis (zygomycosis). Clin Infect Dis. 2012;54 Suppl 1:S55-60. doi: 10.1093/cid/cir868.

11. Rickerts V, Mousset S, Lambrecht E, Tintelnot K, Schwerdtfeger $\mathrm{R}$, Presterl $\mathrm{E}$, et al. Comparison of histopathological analysis, culture, and polymerase chain reaction assays to detect invasive mold infections from biopsy specimens. Clin Infect Dis. 2007;44(8):1078-83. doi: 10.1086/512812.

12. Voigt K, Cigelnik E, O'Donnell K. Phylogeny and PCR identification of clinically important Zygomycetes based on nuclear ribosomal-DNA sequence data. J Clin Microbiol. 1999;37(12):3957-64.

13. Piancastelli C, Ghidini F, Donofrio G, Jottini S, Taddei S, Cavirani $\mathrm{S}$, et al. Isolation and characterization of a strain of Lichtheimia corymbifera (ex Absidia corymbifera) from a case of bovine abortion. Reprod Biol Endocrinol. 2009;7:138. doi: 10.1186/1477-7827-7-138.

14. Paramythiotou E, Frantzeskaki F, Flevari A, Armaganidis A, Dimopoulos G. Invasive fungal infections in the ICU: how to approach, how to treat. Molecules. 2014;19(1):1085-119. doi: 10.3390/molecules19011085.

15. Bala K, Chander J, Handa U, Punia RS, Attri AK. A prospective study of mucormycosis in north India: experience from a tertiary care hospital. Med Mycol. 2015;53(3):248-57. doi: 10.1093/mmy/myu086.

16. Richardson M. The ecology of the Zygomycetes and its impact on environmental exposure. Clin Microbiol Infect. 2009;15 
Suppl 5:2-9. doi: 10.1111/j.1469-0691.2009.02972.x.

17. Park YJ, Choi YK, Min BR. PCR-DGGE and PCR-RFLP analysis of the internal transcribed spacer (ITS) of ribosomal DNA in the genus Rhizopus. J Microbiol. 2003;41(2):157-60.

18. Chakrabarti A, Ghosh A, Prasad GS, David JK, Gupta S, Das $A$, et al. Apophysomyces elegans: an emerging zygomycete in India. J Clin Microbiol. 2003;41(2):783-8.

19. Nyilasi I, Papp T, Csernetics A, Krizsan K, Nagy E, Vagvolgyi C. High-affinity iron permease (FTR1) gene sequence-based molecular identification of clinically important Zygomycetes. Clin Microbiol Infect. 2008;14(4):393-7. doi: 10.1111/j.1469- 0691.2007.01932.x.

20. Shakeri K, Sadeghi M, Deris F, Amani S, Teimouri F, Zebardast $\mathrm{N}$. Evaluation of bacterial and fungal contamination in equipments used in ladies and gentleman barbers in Shahrekord, 2009. Journal of Shahrekord Uuniversity of Medical Sciences. 2011;13(4):102-7.

21. Hoshyar F, Kabiri N, Taghipoor S, Ganji F. Study of fungal infection frequency in libraries affiliated with Shahrekord University of Medical Sciences in 2013. Journal of Shahrekord Uuniversity of Medical Sciences. 2014;16(4):39-45. 